\title{
Speziell-relativistische Auswertung einer Variante der projektiven Relativitätstheorie
}

\author{
Von Ernst Schmutzer \\ Aus dem Theoretisch-Physikalischen Institut der Universität Jena \\ (Z. Naturforschg. 14 a, 486-488 [1959] ; eingegangen am 2. Januar 1959)
}

\begin{abstract}
Um den physikalischen Inhalt der projektiven Relativitätstheorie besser zu erkennen, deren Feldgleichungen nur schwer in konkreten Fällen exakt zu lösen sind, wird diese Theorie auf den Fall des Mгккошsкі-Raumes spezialisiert. Die entstehenden Feldgleichungen sind einer nichtlinearen Elektrodynamik äquivalent und führen deshalb auf die bekannten nichtlinearen Effekte. Insbesondere konvergiert die Feldenergie einer geladenen Punktsingularität, wobei in eine gewisse „Grundlänge“ die Gravitationskonstante eingeht.
\end{abstract}

\section{$\S 1$. Allgemeine speziell-relativistische Theorie}

In diesem Abschnitt spezialisieren wir die allgemeinen projektiv-relativistischen Feldgleichungen und Erhaltungssätze früherer Arbeiten ${ }^{1}$. Als Koordinaten verwenden wir die Minkowski-Koordinaten $x_{1}=x, x_{2}=y, x_{3}=z, x_{4}=i c t$. Im wesentlichen gebrauchen wir dieselbe Symbolik wie in den früheren Arbeiten. Lateinische Indizes laufen also von $1-4$, griechische von $1-5$. Insbesondere bedeuten:

$\gamma$
$\varkappa=6 \pi \gamma / c^{4}$
$A_{i}$
$H_{i j}$
$F_{i j}$
$J$

$j_{k} \quad$ Viererstromdichte,

$\Theta_{i j} \quad$ Energietensor des mechanischen Materieanteils,

$E_{i j} \quad$ Minкоwsкi-Tensor,

$J_{i j} \quad$ Energietensor des $J$-Feldes,

$\stackrel{4}{G}$ 4-dimensionaler Krümmungsskalar.

Zwischen diesen Größen bestehen die folgenden Relationen:

$$
\begin{aligned}
F_{i j} & =\frac{J^{3}}{(2 \varkappa)^{3 / 2}} H_{i j}, \\
E_{i j} & =H_{i m} F_{m j}+\frac{\delta_{i j}}{4} H_{m n} F_{m n}, \\
J_{i j} & =-\frac{1}{\varkappa \sqrt{ } 2 \varkappa}\left(J,{ }_{i},{ }_{j}-\delta_{i j} J,,_{m}, m\right), \\
K & =\frac{1}{S^{2}} T_{\mu \nu} X^{\mu} X^{\nu}, \\
T & =T_{\mu^{\mu}} .
\end{aligned}
$$

1 E. Schmutzer, Z. Phys. 149, 329 [1957], 154, 312 [1959]; Wiss. Z. d. Univ. Jena, Jahrg. 1959, H. 1 (naturw. Reihe);
Die Bewegungsgleichung einer mit der Ladung $e$ versehenen Punktmasse $m$ lautet in dieser spezialisierten Form:

$$
\frac{\mathrm{d}}{\mathrm{d} t}\left(m \frac{\mathrm{d} x_{j}}{\mathrm{~d} t}\right)= \pm \frac{e}{c} H_{j i} \frac{\mathrm{d} x_{i}}{\mathrm{~d} t}+\frac{e^{2}}{m c^{2}} \frac{J, j}{J^{3}} .
$$

Eine derartige geladene Masse ändert sich im J-Feld nach dem Gesetz:

$$
\begin{array}{r}
\stackrel{5}{m_{0}}\left\{\left(1+e^{2} / m_{0}^{2} c^{4} J^{2}\right) /\left(1-v^{2} / c^{2}\right)\right\}^{1 / 2} \\
\left.\stackrel{5}{m_{0}}=\text { const }\right) .
\end{array}
$$

Die Feldgleichungen ergeben sich leicht zu:

$$
\begin{aligned}
F_{m j, j} & =\frac{1}{c} j_{m}, \\
H_{i j} & =A_{j, i}-A_{i, j}, \\
J_{, i, i} & =\frac{\varkappa^{3 / 2}}{\sqrt{ } 2} H_{i j} F_{i j}+\varkappa J K-\frac{\varkappa T J}{3} .
\end{aligned}
$$

Die Gravitationsgleichung wurde dabei in üblicher Weise als näherungsweise erfüllt angesehen. Die Erhaltungssätze lassen sich schreiben:

$$
\left(E_{m}{ }^{n}+\Theta_{m}{ }^{n}\right) ; n=-\frac{1}{(2 \varkappa)^{3 / 2}} \stackrel{4}{G} J,_{m}
$$

(Energie-Impuls-Satz in kovarianter Form),

$$
\boldsymbol{j}_{k, k}=0
$$

(Kontinuitätsgleichung).

Gl. (11) geht für den speziell-relativistischen Fall wegen des Zusammenhanges :

$$
\stackrel{4}{G}=-\frac{3(2 \varkappa)^{3 / 2}}{4 J} F_{i j} H^{i j}-2 \varkappa K
$$

über in

$$
\left(E_{m n}+\Theta_{m n}\right),{ }_{n}=\frac{3}{4 J} J,{ }_{m} H_{i j} F_{i j}+\frac{1}{\sqrt{2} \varkappa} K J,{ }_{m} .
$$

ausführlich in Habil.-Schrift, Jena 1958 (in diesen Arbeiten weitere Literaturzitate). 
Es gilt somit auch im allgemeinen speziell-relativistischen Fall nicht mehr ein üblicher Energie-ImpulsErhaltungssatz. Nur für ein statisches $J$-Feld entsteht für die 4. Komponente die gewohnte Form:

$$
\left(E_{4 n}+\Theta_{4 n}\right),{ }_{n}=.
$$

Als die für die Erhaltung maßgebliche Energiedichte hat man also anzusprechen

$$
u=E_{44}+\Theta_{44} .
$$

In Vektorschreibweise lauten die elektromagnetischen Feldgleichungen, wenn wir in üblicher Weise $F_{m j}$ aus $\mathfrak{S}$ und $\mathfrak{D}$ und $H_{m j}$ aus $\mathfrak{B}$ und $\mathfrak{E}$ aufgebaut denken:

$$
\begin{aligned}
& \operatorname{rot} \mathfrak{S}=\frac{1}{c}\left(\frac{\partial \mathfrak{D}}{\partial t}+\mathfrak{i}\right), \\
& \operatorname{div} \mathfrak{D}=\varrho, \\
& \operatorname{rot} \mathfrak{E}=-\frac{1}{c} \frac{\partial \mathfrak{B}}{\partial t}, \\
& \operatorname{div} \mathfrak{B}=0 .
\end{aligned}
$$

Dabei besteht der Zusammenhang:

$$
(\mathfrak{i})=\left(j_{1}, j_{2}, j_{3}\right), \quad(\varrho)=\frac{1}{i c}\left(j_{4}\right) .
$$

Die elektromagnetische Energiedichte lautet

$$
u^{\mathrm{E}}=\frac{1}{2}(\mathfrak{E} \mathfrak{D}+\mathfrak{S} \mathfrak{B}),
$$

während die Materialgleichungen die Form annehmen:

$$
\mathfrak{S}=\frac{J^{3}}{(2 \varkappa)^{3 / 2}} \mathfrak{B}, \quad \mathfrak{D}=\frac{J^{3}}{(2 \varkappa)^{3 / 2}} \mathfrak{F} .
$$

Für den Fall abwesender mechanischer Materieform, also $\Theta_{m n}=0, K=0, j_{i}=0$, lautet als Energiebilanz Gl. (15) :

$$
\begin{array}{r}
c \operatorname{div}(\mathfrak{F} \times \mathfrak{S})+\frac{1}{2} \frac{\partial}{\partial t}(\mathfrak{F} \mathfrak{D}+\mathfrak{S} \mathfrak{B})+\frac{3}{2 J} \quad(24) \\
(\mathfrak{S} \mathfrak{D}-\mathfrak{S} \mathfrak{B}) \frac{\partial J}{\partial t}=0 .
\end{array}
$$

Dieselbe Beziehung resultiert auch direkt aus den Feldgleichungen.

\section{§ 2. Statisches Feld einer Punktsingularität}

In diesem Spezialfall lauten die zu lösenden Feldgleichungen:

$\operatorname{rot} \mathfrak{S}=0, \quad(25) \operatorname{div} \mathfrak{D}=0,(26) \quad \operatorname{rot} \mathfrak{E}=0$,

$\operatorname{div} \mathfrak{B}=0,(28) \quad \Delta J=\sqrt{2} \varkappa^{3 / 2}(\mathfrak{S} \mathfrak{B}-(\mathfrak{E} \mathfrak{D})$.

Durch Einsetzen überzeugt man sich, daß folgende kugelsymmetrischen Lösungen das Gleichungssystem (25) bis (28) befriedigen:

$$
\begin{gathered}
\mathfrak{D}=e \mathfrak{r} / r^{3} \quad(\text { absolutes Maßsystem }), \\
\mathfrak{E}=\frac{e \mathfrak{r}}{r^{3}} \frac{(2 \varkappa)^{3 / 2}}{J^{3}}(\text { absolutes Maßsystem }), \\
\mathfrak{S}=0, \quad \mathfrak{B}=0 .
\end{gathered}
$$

Gl. (29) geht über in

$$
\begin{aligned}
\Delta J+4 e^{2} \varkappa^{3} / J^{3} r^{4} & =0, \\
\text { oder } \quad(J r)^{\prime \prime}+4 e^{2} \varkappa^{3} /(J r)^{3} & =0 .
\end{aligned}
$$

Nach Kaмke ${ }^{2}$ existieren die beiden Lösungen:

$$
\begin{aligned}
& J_{\mathrm{I}}= \pm \frac{1}{\sqrt{C_{1} r}} \sqrt{4 e^{2} \varkappa^{3}-\left(C_{1} r-C_{2}\right)^{2}}, \\
& J_{\mathrm{II}}= \pm \sqrt{\frac{C}{r^{2}}+\frac{4 e \varkappa^{3 / 2}}{r}}
\end{aligned}
$$$$
\text { (C, } C_{1}, C_{2} \text { Integrationskonstanten). }
$$

Aus physikalischen Gründen fordern wir für $r \rightarrow \infty$ Übergang in die MaxweLLsche Theorie, d.h. $J \rightarrow$ const. Deshalb scheidet die zweite angegebene Lösung aus, während aus der ersten Lösung für $r \rightarrow \infty$ folgt:

$$
J_{\mathrm{I}} \rightarrow \pm i \sqrt{C_{1}} \text {. }
$$

Andererseits ergibt sich aus (31) für $r \rightarrow \infty$

$$
J \rightarrow \sqrt{2 \varkappa} \text {, d. h. } C_{1}=-2 \varkappa .
$$

Damit läßt sich dann (35) schreiben:

$$
J= \pm \frac{\sqrt{2 \varkappa}}{r} \sqrt{\left(r+\frac{C_{2}}{2 \varkappa}\right)^{2}-e^{2} \varkappa} .
$$

Für die Energiedichte $u^{\mathrm{E}}$ resultiert im absoluten Maßsystem:

$$
u^{\mathrm{E}}=\frac{e^{2}}{8 \pi r^{4}}\left[\left(1+\frac{C_{2}}{2 \varkappa r}\right)^{2}-\frac{e^{2} \varkappa}{r^{2}}\right]^{-3 / 2},
$$

woraus für die gesamte Feldenergie im absoluten Maßsystem folgt:

$$
U=4 \pi \int_{0}^{\infty} u^{\mathrm{E}} r^{2} \mathrm{~d} r=\frac{e^{2}}{2} \int_{0}^{\infty} \frac{1}{r^{2}}\left[\left(1+\frac{C_{2}}{2 \varkappa r}\right)^{2}-\frac{e^{2} \varkappa}{r^{2}}\right]^{-3 / 2} \mathrm{~d} r=\frac{1}{2 \varkappa}\left[\frac{C_{2}}{2 \varkappa}-\sqrt{\frac{C_{2}^{2}}{4 \varkappa^{2}}-e^{2} \varkappa}\right] .
$$

\footnotetext{
2 E. Kамке, Differentialgleichungen, 1. Bd., Akadem. Verlagsgesellschaft Geest u. Portig, Leipzig 1944, S. 590.
} 
In dieser Beziehung tritt noch die Integrationskonstante $C_{2}$ auf. Aus physikalischen Gründen muß $C_{2}{ }^{2} \geqq 4 e^{2} \varkappa^{3}$ sein. Für $C_{2} \rightarrow \infty$ geht $U \rightarrow 0$. Ein anderer ausgezeichneter Wert von $C_{2}$ ist

$$
C_{2}=2 \varkappa^{3 / 2} e,
$$

für den $U$ maximal wird. $U$ liegt also im Intervall $0 \leqq U \leqq U_{\text {Max }}$, wobei

$$
U_{\mathrm{Max}}=C_{2} / 4 \varkappa^{2}=e / 2 \sqrt{\varkappa} \text { ist. }
$$

Integriert man über das gesamte Volumen, so findet man für die wahre Ladung $Q$ (dem $\mathfrak{D}$-Feld zugeordnet) und für die freie Ladung $Q^{\text {frei }}$ (dem EF-Feld zugeordnet) bei Anwendung des Gaussschen Satzes das Resultat:

$$
Q=\int \varrho \mathrm{d} V=e \int \delta(\mathrm{r}) \mathrm{d} V=e,
$$

$Q^{\text {frei }}=\int \varrho^{\text {frei }} \mathrm{d} V=\int \operatorname{div} \Leftarrow \mathrm{d} V=\int \mathcal{E} \mathrm{d} f=e$.

Abschließend diskutieren wir des Interesses wegen den Extremfall (42) : Die fiktive Dielektrizitätskonstante hat dann folgenden Verlauf:

$$
\varepsilon=\frac{D}{E}=\frac{J^{3}}{(2 \varkappa)^{3 / 2}}=\left[1+\frac{2 e \sqrt{\varkappa}}{r}\right]^{3 / 2} .
$$

Für $r \rightarrow 0$ geht also $\varepsilon \rightarrow \infty$. J selbst lautet für diesen Fall:

$$
J= \pm \sqrt{2 x} \sqrt{1+\frac{2 e \sqrt{x}}{r}} .
$$

Für $r \rightarrow 0$ haben wir also folgendes Grenzverhalten vorliegen:

$$
J \rightarrow \pm 2 \sqrt{e} x^{3 / 4} / \sqrt{r} .
$$

Rechnen wir für diesen Grenzfall die Feldmasse $M_{\max }=U_{\max } / c^{2}$ aus und führen wir einen fiktiven
Teilchenradius $r_{0}$ („Grundlänge“) ein, so folgt:

$$
M_{\max }=e / 2 c^{2} \sqrt{\varkappa}=e^{2} / c^{2} r_{0},
$$

woraus man findet

$$
r_{0}=2 e \sqrt{\varkappa} .
$$

Durch Elimination von $e$ vermöge $e^{2}=\hbar c / 137$ gewinnt man die Beziehung:

$$
r_{0}=2 \sqrt{\varkappa \hbar c / 137} .
$$

Das Interessante an dieser Formel ist das Auftreten der Gravitationskonstanten, mit der sich bekanntlich in der obigen Weise eine Längeneinheit bilden läßt. Zahlenmäßig entspricht der obige Extremfall hypothetischen Teilchen, die eine extrem große Masse besitzen würden und außerdem instabil wären

$$
\left(M_{\max } \approx 10^{-7} \mathrm{~g} ; \quad r_{0} \approx 10^{-33} \mathrm{~cm}\right) .
$$

Teilchen von der Masse eines Elektrons würde die Wahl $C_{2}=e^{2} \varkappa / 2 m_{\mathrm{el}} c^{2}$ entsprechen. Wegen der Singularitäten von (10) würde sich die Feldmasse zur mechanischen Masse solcher instabiler Teilchen wie $3 / 4$ verhalten.

Abgesehen von einer möglichen Existenz solcher Partikel in der Natur, haben wir hier Konsequenzen aus der projektiven Relativitätstheorie gezogen, um dadurch der Frage der Leistungsfähigkeit dieser Theorie insgesamt näher zu kommen. Vielleicht erscheint auch dadurch die Problematik einer natürlichen Elementarlänge in neuem Licht. Um dem Inhalt der projektiven Relativitätstheorie selbst näher auf die Spur zu kommen, ist es erforderlich, auf der Grundlage der in $\S 1$ entwickelten nichtlinearen Theorie weitere Effekt zu berechnen. Insbesondere soll eine demnächst folgende Arbeit die Streuung von Licht an Licht untersuchen. 